\title{
Curvature Estimation Using Shape-from-Texture
}

\author{
Eraldo Ribeiro and Edwin R. Hancock \\ Department of Computer Science \\ University of York, York, YO10 5DD, UK
}

\begin{abstract}
This paper shows how the apparatus of robust statistics can be used to extract consistent estimates of surface orientation using shapefrom-texture. We make initial estimates of surface orientation by measuring the affine distortion of neighbouring spectral peaks. We show how the initial orientation estimates can be refined using a process of robust smoothing and subsequently used for reliable curvature estimation. We apply the method to a variety of real-world and synthetic imagery. Here it is demonstrated to provide useful estimates of curvature.
\end{abstract}

\section{Introduction}

The recovery of surface shape using texture information is a process that is grounded in psychophysics. Moreover, it has been identified by Marr 11 as being a potentially useful component the $2 \frac{1}{2} \mathrm{D}$ sketch. Stated succinctly the problem is as follows. Given a two dimensional image of a textured surface, how can the three dimensional shape of the viewed object be recovered $2,3,4,5,6]$ ? There are two contributions that deserve special attention. The first of these is the work of Garding [5] who has developed an elegant differential framework for shapefrom-texture. The contribution here is to link the differential geometry of curved surfaces to variations in texture gradient. However, the practical realisation of the method has been confined to the use of artificial structural texture primitives. The second noteworthy contribution is that of Rosenholtz and Malik [6]. This is a frequency domain approach. The aim is to recover local surface orientation parameters which minimise a back-projection error which measures the residual texture gradient. However, the method relies on numerical optimisation and is only demonstrated on rather artificial imagery.

One of the criticisms that can be levelled at existing shape-from-texture methods, is their failure to deliver information of sufficient acuity for reliable surface analysis. In a recent paper, we have addressed the analogous problem of extracting useful topographic information from shape-from-shading [7. Here we have shown how the apparatus of robust statistics can be used to refine an initially noisy field of surface normal estimates to recover reliable information concerning surface topography. The aim in this paper is to return to the shape-from-texture problem and show how the initial estimates of surface orientation delivered by local spectral analysis can be refined and used for subsequent curvature analysis. It must be stressed that recovery of shape from texture is more challenging than 
the recovery of shape from shading since the process is not constrained by the physics of light.

The recovery of a dense field of tangent plane orientations is a two-step process. The first step is to make an initial estimate of the local surface orientation. Here we use the eigenvectors of the affine distortion matrix for corresponding spectral peaks to estimate the slant and tilt directions for local tangent planes. Once the initial surface normal estimates are to hand, the second step is to improve the consistency of the orientation field through the use of local contextual information. Conventionally, this second step is realised by smoothing the estimated directions of the surface normals through a process of local averaging. Here we adopt a more elaborate smoothing method which has proved successful in the shape-from-shading domain. We use robust error kernels rather than quadratic smoothness penalties to improve the organisation of the needle map. This allows us to preserve fine surface detail whilst removing the effects of local noise. We experiment with the new shape-from-texture on demanding real world images of curved texture surface. Here the method produces qualitatively good results.

\section{Geometric Modelling}

We commence by reviewing the projective geometry for the perspective transformation of points on a plane. Specifically, we are interested in the perspective transformation between the object-centred co-ordinates of the points on the texture surface and the viewer-centred co-ordinates of the corresponding points on the image plane. To be more formal, suppose that the texture surface is a distance $h$ from the camera which has focal length $f<0$. Consider two corresponding points. The point with co-ordinates $\mathbf{X}_{\mathbf{t}}=\left(x_{t}, y_{t}, z_{t}\right)^{T}$ lies on the texture surface while the corresponding point on the image plane has co-ordinates $\mathbf{X}_{\mathbf{i}}=\left(x_{i}, y_{i}, f\right)^{T}$. We represent the orientation of the viewed texture surface in the image plane co-ordinate system using the slant $\sigma$ and tilt $\tau$ angles. For a given plane, the slant is the angle between viewer line of sight and the normal vector of the plane. The tilt is the angle of rotation of the normal vector to the texture plane around the line of sight axis. Furthermore, since we regard the texture as being "painted" on the texture surface, the texture height $z_{t}$ is always equal to zero. With these ingredients the perspective transformation between the texture-surface and image co-ordinate systems is given in matrix form by

$$
\left[\begin{array}{l}
x_{i} \\
y_{i} \\
z_{i}
\end{array}\right]=\frac{f}{h-x_{t} \sin \sigma} \times\left\{\left[\begin{array}{ccc}
\cos \sigma \cos \tau & -\sin \tau & \sin \sigma \cos \tau \\
\cos \sigma \sin \tau & \cos \tau & \sin \sigma \sin \tau \\
-\sin \sigma & 0 & 1
\end{array}\right]\left[\begin{array}{l}
x_{t} \\
y_{t} \\
z_{t}
\end{array}\right]+\left[\begin{array}{l}
0 \\
0 \\
h
\end{array}\right]\right\}
$$

The first term inside the curly braces represents the rotation of the local texture plane in slant and tilt. The second term represents the displacement of the rotated plane along the optic axis. Finally, the multiplying term outside the braces represents the non-linear foreshortening in the slant direction. When expressed in this way, $z_{i}$ is always equal to $f$ since the image is formed at the 
focal plane of the camera. This transformation can be represented using the shorthand $\left(x_{i}, y_{i}\right)^{T}=T_{p}\left(x_{t}, y_{t}\right)^{T}$, where $T_{p}$ is a $2 \times 2$ transformation matrix [3].

Unfortunately, the non-linear nature of the perspective transformation makes the Fourier domain analysis of the texture somewhat intractable. To overcome this difficulty it is usual to use a linear approximation of the perspective projection 23 . To proceed we follow the bulk of the literature on shape-from-texture and use a locally affine approximation to the perspective transformation [2]. Let $X_{o i}=\left(x o_{t}, y o_{t}, h\right)^{T}$ be the location of the origin or expansion point for the local co-ordinate system of the affine transformation. This origin projects to the point $\left(x o_{i}, y o_{i}, f\right)$ on the image plane. We denote the local coordinate system on the image plane by $\mathbf{X}_{\mathbf{i}}^{\prime}=\left(x_{i}^{\prime}, y_{i}^{\prime}, f\right)$ where $x_{i}=x_{i}^{\prime}+x o_{i}$ and $y_{i}=y_{i}^{\prime}+y o_{i}$. The affine approximation is given by $T_{A}\left(\mathbf{X}_{o i}\right)=J\left(\mathbf{X}_{o i}\right)=\left.J\left(T_{p} \mathbf{X}_{\mathbf{t}}\right)\right|_{\left(\mathbf{X}_{\mathbf{i}}^{\prime}=\mathbf{0}\right)}$ where $J\left(\mathbf{X}_{\mathbf{i}}\right)$ is the Jacobian matrix of $\mathbf{X}_{\mathbf{i}}$. Rewriting $T_{A}$ in terms of the slant and tilt angles we have

$$
T_{A}\left(\mathbf{X}_{o i}\right)=\frac{\Omega}{h f \cos \sigma} \times\left[\begin{array}{ll}
x o_{i} \sin \sigma+f \cos \tau \cos \sigma & -f \sin \tau \\
y o_{i} \sin \sigma+f \sin \tau \cos \sigma & f \cos \tau
\end{array}\right]
$$

where $\Omega=f \cos \sigma+\sin \sigma\left(x o_{i} \cos \tau+y o_{i} \sin \tau\right)$. Hence, the affine transformation matrix $T_{A}$ depends only on the expansion point $\left(x o_{i}, y o_{i}\right)$, which is a constant, together with the slant and tilt angles, which are the goal of our analysis.

We now turn our attention to how the frequency content of the local texture plane transforms under local affine geometry. Our starting point is a well known property which relates the effect of an affine transformation in the spatial domain to the Fourier-domain representation of a signal [8]. Suppose that $G($.$) represents$ the Fourier transform of a signal. Furthermore, let $\mathbf{X}$ be a vector of spatial coordinates and let $\mathbf{U}$ be the corresponding vector of frequencies. According to Bracewell et al, the distribution of image-plane frequencies $\mathbf{U}_{\mathbf{t}}$ resulting from the Fourier transform of the affine transformation is given by

$$
G\left(\mathbf{U}_{\mathbf{i}}\right)=\frac{1}{\left|\operatorname{det}\left[T_{A}(X)\right]\right|} G\left[\left(T_{A}(X)^{T}\right)^{-1} \mathbf{U}_{\mathbf{t}}\right]
$$

Applying the Fourier property of Equation 3 to the linearised version of the perspective transformation, the relationship between the texture plane and image spectra is

$$
\mathbf{U}_{\mathbf{i}}=T_{A}(X)^{-T} \mathbf{U}_{\mathbf{t}}
$$

Here, we will consider only the affine distortion in the positions of frequency peaks. In other words we will not consider the distribution of the energy amplitude or phase in our analysis. For practical purposes we will use the local power spectrum to locate the positions of spectral peaks.

\section{Spectral Distortion across the Image Plane}

In this section we show how to make initial estimates of surface orientation using the results presented in the previous Section. To commence, we consider 
the point $S$ on the curved texture surface. Suppose that the neighbourhood of this point can be approximated by a local planar patch. This planar patch undergoes perspective projection onto the image plane. Using the result presented in Section 2 we make a locally affine approximation to this perspective projection. Further suppose we sample the texture projection of the local planar patch at two neighbouring points $A$ and $B$ laying on the image plane. The co-ordinates of the two points are respectively $\mathbf{X}_{A}=(x, y)^{T}$ and $\mathbf{X}_{B}=(x+\Delta x, y+\Delta y)^{T}$ where $\Delta x$ and $\Delta y$ are the image-plane displacements between the two points.

Suppose that the local planar patch on the texture surface has a spectral peak with frequency vector $U_{S}=\left(u_{s}, v_{s}\right)^{T}$. On the image plane, the corresponding frequency vectors for the spectral peaks at the points $X_{A}$ and $X_{B}$ are respectively $U_{A}=\left(u_{A}, v_{A}\right)^{T}$ and $U_{B}=\left(u_{B}, v_{B}\right)^{T}$. Using the Fourier domain affine projection property presented in Section 3, the texture-surface peak frequencies are related to the image plane peak frequencies via the equations $U_{A}=\left(T_{A}\left(X_{A}\right)^{-1}\right)^{T} U_{S}$ and $U_{B}=\left(T_{A}\left(X_{B}\right)^{-1}\right)^{T} U_{S}$, where $T_{A}\left(X_{A}\right)$ is the local affine approximation to the perspective projection of the planar surface patch at the point $A$ and $T_{A}\left(X_{B}\right)$ is the corresponding affine projection matrix at the point $B$. As a result, the frequency vectors for the two corresponding spectral peaks on the image-plane are related to one-another via the affine distortion $U_{B}=\left(T_{A}\left(X_{A}\right) T_{A}\left(X_{B}\right)^{-1}\right)^{T} U_{A}$. As a result, the texture-surface spectral distortion matrix $\Phi=\left(T_{A}\left(X_{A}\right) T_{A}\left(X_{B}\right)^{-1}\right)^{T}$ is a $2 \times 2$ matrix. This matrix relates the affine distortion of the image plane frequency vectors to the 3 -D orientation parameters of the local planar patch on the surface. Substituting for the affine approximation to the perspective transformation from Equation (5), the required matrix is given in terms of the slant and tilt angles as

$$
\Phi=\frac{\Omega(\mathbf{A})}{\Omega^{2}(\mathbf{B})}\left[\begin{array}{cc}
\Omega(\mathbf{A})+\Delta y \sin \sigma \sin \tau & -\Delta y \sin \sigma \cos \tau \\
-\Delta x \sin \sigma \sin \tau & \Omega(\mathbf{A})+\Delta x \sin \sigma \cos \tau
\end{array}\right]
$$

where $\Omega(A)=f \cos \sigma+\sin \sigma(x \cos \tau+y \sin \tau)$ and $\Omega(B)=f \cos \sigma+\sin \sigma \times$ $((x+\Delta x) \cos \tau+(y+\Delta y) \sin \tau)$. The above matrix represents the linear mapping governing the spectral distortion over the image plane. It accounts for distortion of the spectrum sampled at the location $B$ with respect to the sample at the location $A$. Next, we show how to solve directly for the parameters of surface orientation, i.e. the slant and tilt angles, using the eigen-structure of the transformation matrix $\Phi$.

Let us consider the eigenvector equation for the affine distortion matrix $\Phi$, i.e. $\Phi \mathbf{w}(\lambda)=\lambda \mathbf{w}(\lambda)$, where $\lambda=\left(\lambda_{1}, \lambda_{2}\right)$ are the eigenvalues of the transformation $\Phi$ and $\mathbf{w}(\lambda)$ are the corresponding eigenvalues. We can directly determine the tilt angle from the direction of the eigenvector associated with the eigenvalue $\lambda_{1}$. It can be shown that the direction of the leading eigenvector can be used to estimate the tilt direction using the relation

$$
\tau=\arctan \left(\frac{\mathbf{w}_{y}\left(\lambda_{1}\right)}{\mathbf{w}_{x}\left(\lambda_{1}\right)}\right)
$$


Once the tilt angle has been obtained, the slant angle can be recovered using the second eigenvalue via the relationship

$$
\sigma=\arctan \left[\frac{f\left(\lambda_{2}-1\right)}{\left(y\left(1-\lambda_{2}\right)-\lambda \Delta y\right) \sin \tau+\left(x\left(1-\lambda_{2}\right)-\lambda_{2} \Delta x\right) \cos \tau}\right]
$$

The first step in orientation recovery is to estimate the affine distortion matrix which represents the transformation between different local texture regions on the image plane. These image texture regions are assumed to belong to single local planar patch on the curved texture surface. Suppose that $U_{1}=\left(u_{1}, v_{1}\right)^{T}$ represents a spectral peak estimated at the point with co-ordinates $\left(x_{1}, y_{1}\right)$ on the image plane. Further, suppose that $U_{2}=\left(u_{2}, v_{2}\right)^{T}$ is the corresponding spectral peak at the point $\left(x_{2}, y_{2}\right)$. Under the affine model presented in Section 3 , the two peaks are related via the equation $\mathbf{U}_{2}=\Phi \mathbf{U}_{1}$. Consequently, the local estimate of the affine spectral distortion matrix is $\Phi=\left(\mathbf{U}_{1}^{T}\right)^{-1} \mathbf{U}_{2}$. We only make use of the most energetic peaks appearing in the power spectrum. That is to say we do not consider the detailed distribution of frequencies. Our method requires that we supply correspondences between spectral peaks so that the affine distortion matrices can be estimated. We use the energy amplitude of the peaks is establish the required correspondences. We order the peaks according to their energy amplitude. The ordering of the amplitudes of peaks at different image locations determines the required spectral correspondence. After estimating the affine transform between two local spectral peaks we can directly apply the eigenvector analysis described above to estimate the tilt and the slant angles.

\section{Robust Smoothing of the Needle-Map}

The orientation estimates returned by the new shape-from-texture method are likely to be noisy and inconsistent when viewed from the perspective of local smoothness. In order to improve the consistency of our needle map, and hence the surface shape description, we employ an iterative smoothing process to update the estimated normal vectors. However, in order to avoid the over-smoothing of local surface detail associated with high curvature features, we use a robust smoothing method. Rather than using a quadratic penalty, the error function uses robust error kernels. to gauge the effect of the smoothness error. The reason for this is that the quadratic penalty grows indefinitely with increasing smoothness error. This can have the undesirable effect of over-smoothing genuine surface detail. Examples of such surface structures include ridge and ravine structures. By contrast, robust error kernels moderate the effects of smoothing over regions of genuine surface detail and allow a more faithful topographic representation to be recovered [7].

We choose to use the robust smoothness penalty

$$
I=\iint\left\{\left(\rho_{\sigma}\left(\left\|\frac{\partial \mathbf{n}}{\partial x}\right\|\right)+\rho_{\sigma}\left(\left\|\frac{\partial \mathbf{n}}{\partial y}\right\|\right)\right)\right\} d x d y
$$


In the above measure, $\rho_{\sigma}(\eta)$ is the robust error kernel used to gauge the local consistency of the needle-map or field of surface normals. The argument of the kernel $\eta$ is the measured error and the parameter $\sigma$ controls the width of the kernel. It is important to note the robust-error kernels are applied separately to the magnitudes of the derivatives of the needle-map in the $x$ and $y$ directions. Applying variational calculus the update equation for the surface normals which minimises the smoothness penalty is

$$
\begin{aligned}
\mathbf{n}_{i, j}^{(k+1)}= & \left\|\frac{\partial \mathbf{n}_{i, j}^{(k)}}{\partial x}\right\|^{-1}\left[\frac{\partial}{\partial x}\left(\rho_{\sigma}^{\prime}\left(\left\|\frac{\partial \mathbf{n}_{i, j}^{(k)}}{\partial x}\right\|\right)\right)+\rho_{\sigma}^{\prime}\left(\left\|\frac{\partial \mathbf{n}_{i, j}^{(k)}}{\partial x}\right\|\right) \times\right. \\
& \left.\left(\mathbf{n}_{i+1, j}^{(k)}+\mathbf{n}_{i-1, j}^{(k)}-\left\|\frac{\partial \mathbf{n}_{i, j}^{(k)}}{\partial x}\right\|^{-2}\left(\frac{\partial \mathbf{n}_{i, j}^{(k)}}{\partial x} \cdot \frac{\partial^{2} \mathbf{n}_{i, j}^{(k)}}{\partial x^{2}}\right) \frac{\partial \mathbf{n}_{i, j}^{(k)}}{\partial x}\right)\right] \\
+ & \left\|\frac{\partial \mathbf{n}_{i, j}^{(k)}}{\partial y}\right\|^{-1}\left[\frac{\partial}{\partial y}\left(\rho_{\sigma}^{\prime}\left(\left\|\frac{\partial \mathbf{n}_{i, j}^{(k)}}{\partial y}\right\|\right)\right)+\rho_{\sigma}^{\prime}\left(\left\|\frac{\partial \mathbf{n}_{i, j}^{(k)}}{\partial y}\right\|\right) \times\right. \\
& \left.\left(\mathbf{n}_{i, j+1}^{(k)}+\mathbf{n}_{i, j-1}^{(k)}-\left\|\frac{\partial \mathbf{n}_{i, j}^{(k)}}{\partial y}\right\|^{-2}\left(\frac{\partial \mathbf{n}_{i, j}^{(k)}}{\partial y} \cdot \frac{\partial^{2} \mathbf{n}_{i, j}^{(k)}}{\partial y^{2}}\right) \frac{\partial \mathbf{n}_{i, j}^{(k)}}{\partial y}\right)\right]
\end{aligned}
$$

where $\mathbf{n}_{i, j}^{(k)}$ is the estimated surface normal at the pixel with row index $i$ and column index $j$ at iteration $k$ of the smoothing process.

As stated in Equation (28), the smoothing process is entirely general. Any robust error kernel $\rho_{\sigma}(\eta)$ can be inserted into the above result to yield a needle-map smoothing process. However, it must be stressed that performance is critically determined by the choice of error-kernel. We have found the most effective error kernel to be the log-cosh sigmoidal-derivative M-estimator. The kernel has the functional form

$$
\rho_{\sigma}(\eta)=\frac{\sigma}{\pi} \log \cosh \left(\frac{\pi \eta}{\sigma}\right)
$$

\section{Curvature Estimation}

Once the smoothed needle-map is to hand, then we can use the surface normals to estimate curvature. In our experiments, we have investigated the quality of the shape-index of Koenderink and Van Doorn as scale-invariant measure of surface topography.

The differential structure of a surface is captured by the Hessian matrix, which may be written in terms of surface normals as

$$
\mathcal{H}=\left(\begin{array}{ll}
\left(\frac{\partial \mathbf{n}}{\partial x}\right)_{x} & \left(\frac{\partial \mathbf{n}}{\partial x}\right)_{y} \\
\left(\frac{\partial \mathbf{n}}{\partial y}\right)_{x} & \left(\frac{\partial \mathbf{n}}{\partial y}\right)_{y}
\end{array}\right)
$$


where $(\cdots)_{x}$ and $(\cdots)_{y}$ denote the $x$ and $y$ components of the parenthesised vector respectively. The eigenvalues of the Hessian matrix, found by solving the equation $|\mathcal{H}-\kappa \mathbf{I}|=0$, are the principal curvatures of the surface, denoted $\kappa_{1,2}$. The shape index is defined in terms of the principal curvatures

$$
\phi=\frac{2}{\pi} \arctan \frac{\kappa_{2}+\kappa_{1}}{\kappa_{2}-\kappa_{1}} \quad \kappa_{1} \geq \kappa_{2}
$$

The magnitude of the curvature is measured by the curvedness $\kappa=\frac{2}{\pi} \sqrt{\kappa_{1}^{2}+\kappa_{2}^{2}}$.

\section{Experiments with Curved Surfaces}

We have experimented with both synthetic surfaces with known ground truth and real-world images. The former are used to assess the accuracy of the method, while we use the latter to demonstrate the practical utility of the method.

In Figure 1 we show scatter plots of the ground-truth and the estimated slant angle, tilt angle and shape-index for a synthetic curved textured surface. In each case there is a clear regression line. The parameter of our spectral distortion method is the distance between the points used to estimate the affine distortion matrix on the image plane. If this distance is too small then the affine distortion becomes undetectable. If, on the other hand, the distance is too big then we sample changes in surface orientation rather than perspective foreshortening. In Figure 2 and 3 for the smoothed and unsmoothed needle maps we show a series of scatter plots of the shape-index returned using different values of the interpoint distance $r$ (which is listed in the figure caption). The straight-line plotted through the data is least-squares regression fitted to the data; listed in the figure caption are the values of the linear regression coefficient $\mu$ fitted to the data. The main feature to note from these plots is that in the case of the smoothed version, the slope of the regression line is closer to unit, i.e. the estimated shapeindex is in better agreement with the ground truth. In Figure 4 for the smoothed (a) and unsmoothed (b) needle-maps we plot the linear regression coefficients extracted from the scatter plots as a function of the interpoint distances. If the shape-index measurements are unbiased then the linear regression co-efficient
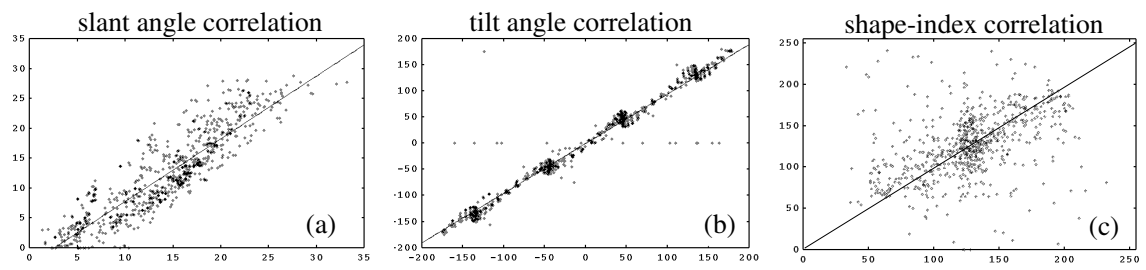

Fig. 1. Scatter Correlation Plots. (a) Slant Angle Correlation; (b) Tilt Angle Correlation; (c) Shape Index Correlation. 

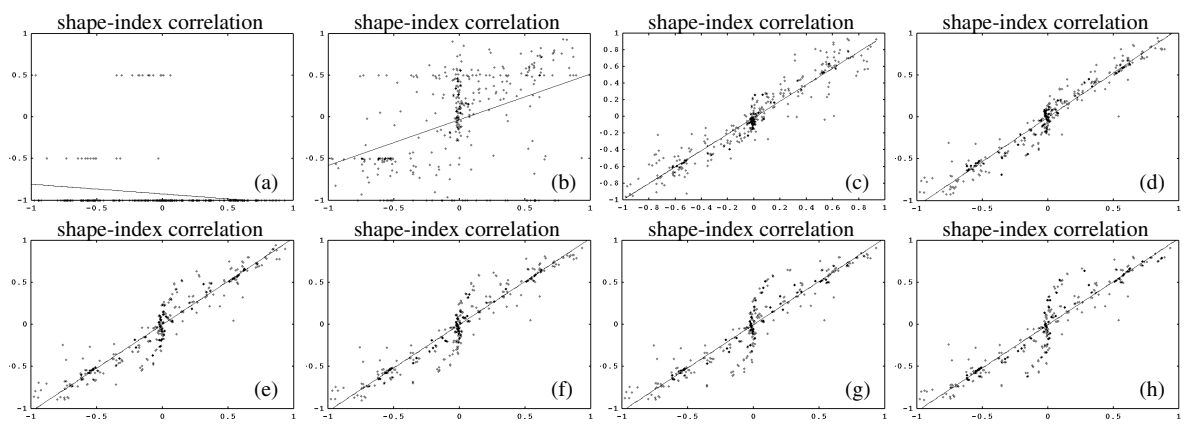

Fig. 2. Effect of neighborhood radius on shape-index for smoothed needle maps: (a) $\mathrm{r}$ $=2 \mu=-0.16$; (b) $\mathrm{r}=4 \mu=0.41$; (c) $\mathrm{r}=8 \mu=0.80$; (d) $\mathrm{r}=16 \mu=0.97$; (e) $\mathrm{r}=$ $32 \mu=0.94$; (f) $\mathrm{r}=48 \mu=0.93$; (g) $\mathrm{r}=64 \mu=0.90$; (h) $\mathrm{r}=80 \mu=0.85$. Where $\mu$ is the linear correlation coefficient and $\mathrm{r}$ is the radius between two patches.

should be unity. The main feature to note is that there is a critical value of the distance which results in a maximum value of the regression co-efficient. For the smoothed needle-maps, the linear regression coefficient is closest to unity (0.97) when the interpoint distance is $r=16$ pixels; this represents an improvement over the initial unsmoothed value of $\mu=0.51$. For the unsmoothed needle-maps, the best regression coefficient (0.84) is obtained when $r=48$ pixels; here the corresponding smoothed value is $\mu=0.93$..

Finally, we experiment with real world textured surfaces. We have generated the images used in this study by moulding regularly textured sheets into curved surfaces. The images used in this study are shown in the first column of Figure 5

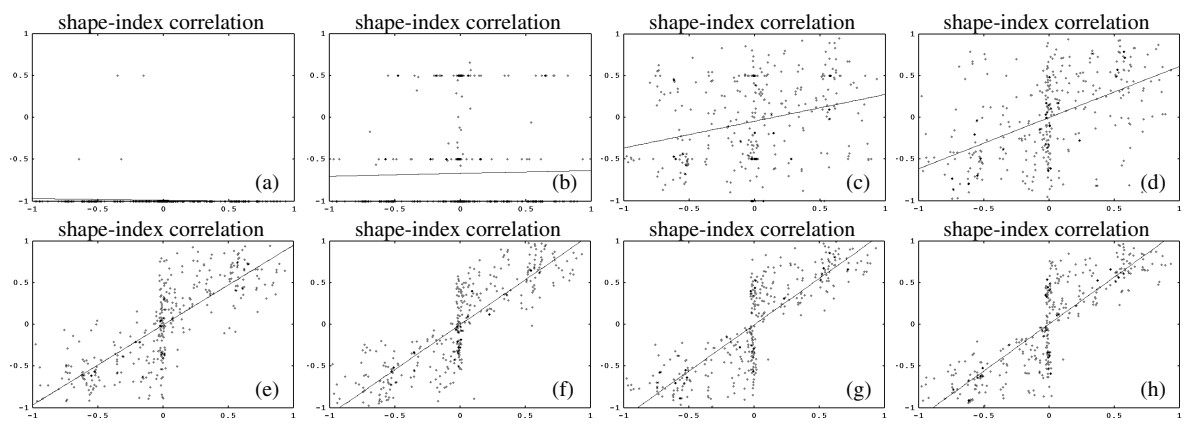

Fig. 3. Effect of neighborhood radius on shape-index for un-smoothed needle-mapes: (a) $\mathrm{r}=2 \mu=-0.07$; (b) $\mathrm{r}=4 \mu=0.27$; (c) $\mathrm{r}=8 \mu=0.26$; (d) $\mathrm{r}=16 \mu=0.51$; (e) $\mathrm{r}=32 \mu=0.79$; (f) $\mathrm{r}=48 \mu=0.84$; (g) $\mathrm{r}=64 \mu=0.80$; (h) $\mathrm{r}=80 \mu=0.72$. Where $\mu$ is the linear correlation coefficient and $\mathrm{r}$ is the radius between two patches. 
Shape-index correlation x Neighbourhood radius Shape-index correlation x Neighbourhood radius
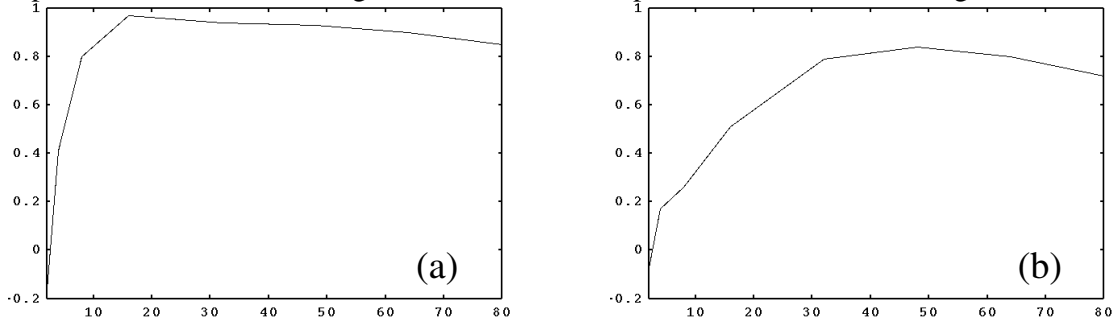

Fig. 4. Plot of the shape index correlation in terms of the neighborhood radius. (a) Smoothed needle map; (b) Non-Smoothed needle map.
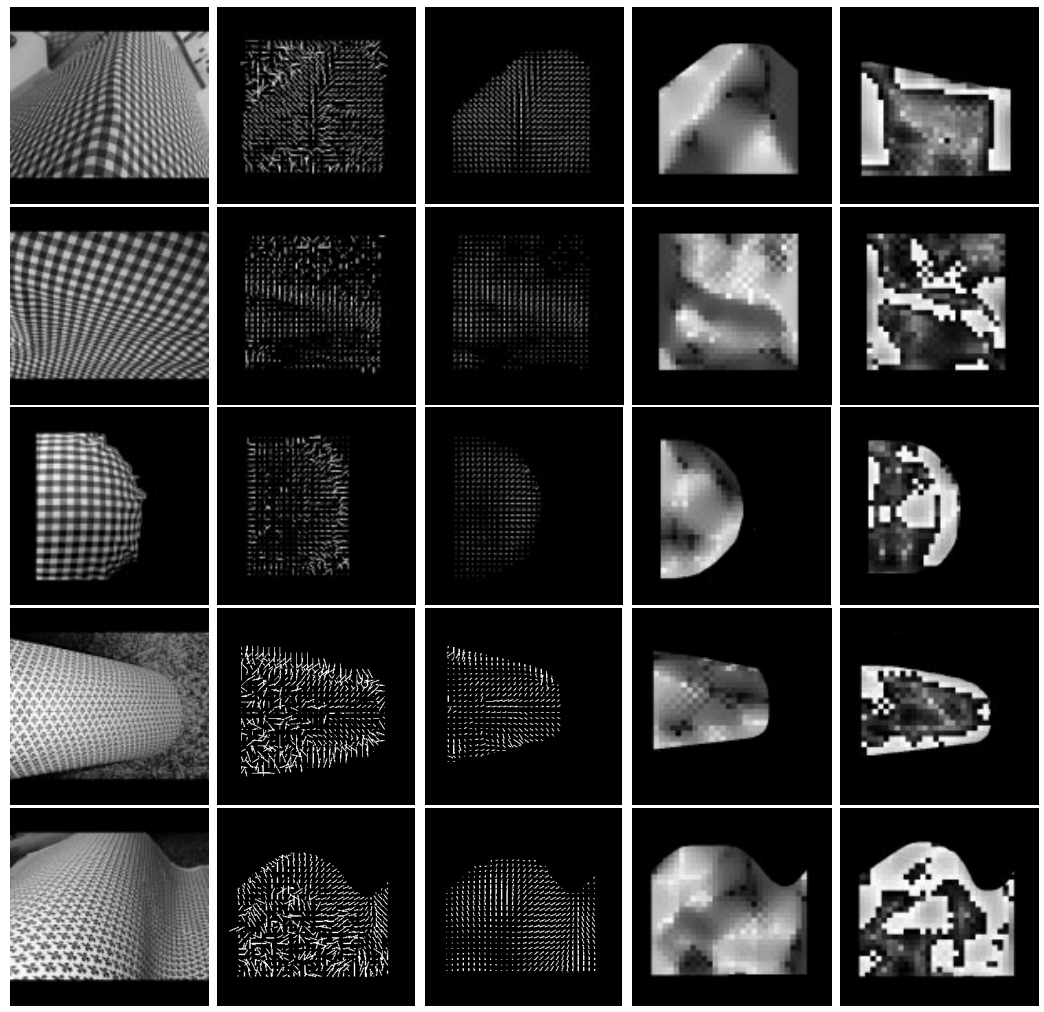

Fig. 5. Real curved surfaces. (a) original image; (b) recovered needle map; (c) Smoothed needle map; (d) Shape index map; (e) Curvedness map. 
There are two sets of images. The first group have been created by placing a table-cloth with a rectangular texture pattern on top of surfaces of various shapes. The second group of images have been created by bending a sheet of wrapping paper into various tubular shapes.

The remaining columns of Figure 5, from left to right, show the initial needlemap, the final smoothed needle-map, the estimated shape-index and the estimated curvedness. In the case of this real world data, the initial needle maps are more noisy and disorganised than their synthetic counterparts. However, there are clear regions of needle-map consistency. When robust smoothing is applied to the initial needle maps, then there is a significant improvement in the directional consistency of the needle directions.

\section{Conclusions}

We have presented a new method for improving the directional consistency of local tangent planes to textured surfaces. The method commences by finding affine distortion matrices for neighbouring points on the image plane. The directions of the eigenvalues of the affine distortion matrices can be used to make closed form estimates of the slant and tilt directions. The initial orientation estimates are iteratively refined using a robust smoothing technique to produce a needle map of improved consistency.

The method is demonstrated on both synthetic imagery with known ground truth and on real-world images of man-made textured surfaces. The method proves useful in the analysis of both planar and curved surfaces. Moreover, the extracted needle maps can be used to make reliable estimates of surface curvature information.

\section{References}

1. David Marr. Vision: A computational investigation into the human representation and processing of visual information. Freeman, 1982.

2. John Krumm and Steve A. Shafer. Shape from periodic texture using spectrogram. In IEEE Conference on Computer Vision and Pattern Recognition, pages 284-289, 1992.

3. B.J. Super and A.C. Bovik. Planar surface orientation from texture spatial frequencies. Pattern Recognition, 28(5):729-743, 1995.

4. John R. Kender. Shape from texture: an aggregation transform that maps a class of texture into surface orientation. In 6th IJCAI, Tokyo, pages 475-480, 1979.

5. Jonas Garding. Shape from texture for smooth curved surfaces in perspective projection. J. of Mathematical Imaging and Vision, 2:329-352, 1992.

6. J. Malik and R. Rosenholtz. Recovering surface curvature and orientation from texture distortion: a least squares algorithm and sensitive analysis. Lectures Notes in Computer Science - ECCV'94, 800:353-364, 1994.

7. P. L. Worthington and E.R. Hancock. Needle map recovery using robust regularizers. Image and Vision Computing, 17(8):545-559, 1998.

8. R.N. Bracewell, K.-Y. Chang, A.K. Jha, and Y.-H. Wang. Affine theorem for twodimensional fourier transform. Electronics Letters, 29(3):304, 1993. 